\title{
Automatic equipment for testing of complex multiparametric intelligent devices
}

\author{
A. Gorchakovsky \\ Institute of engineering physic and radio electronics, \\ Siberian federal university \\ Krasnoyarsk, Russian Federation \\ e-mail: vigetch@list.ru
}

\section{A. Kamyshnikov}

Institute of engineering physic and radio electronics, Siberian federal university

Krasnoyarsk, Russian Federation

e-mail: AL85241@yandex.ru

\section{A. Mishurov}

Institute of engineering physic and radio electronics, Siberian federal university

Krasnoyarsk, Russian Federation

e-mail: amishurov@sfu-kras.ru

\author{
V. Sukhotin \\ Military engineering institute, \\ Siberian federal university \\ Krasnoyarsk, Russian Federation \\ e-mail: vsuhotin@sfu-kras
}

\begin{abstract}
This paper describes the structure and principles of automatic equipment for testing of complex multiparametric intelligent devices.
\end{abstract}

Keywords - Automated test equipment, intelligent devices, testing of complex multiparametric devices, ATE, HTE.

\section{INTRODUCTION}

In the process of development, production, intermediate and acceptance testing of complex high-technology multiparametric intelligent devices it is required to carry out multiple tests of components and systems in accordance with technical requirements [1]. Monitoring and validation of high-technology equipment is one of the most important tasks arising in the process of its production [2]. The testing procedure may take up to several months.

The increasing complexity and growing functionality of the products lead to a significant increase in time spent on carrying out acceptance tests and the costs associated with them. Experience shows that traditional methods of testing based on the use of various measuring devices in manual mode not only lead to increased duration and cost of the testing process but also significantly increase the probability

\author{
V. Evstratko \\ Institute of engineering physic and radio electronics, \\ Siberian federal university \\ Krasnoyarsk, Russian Federation \\ e-mail: evstrafly@list.ru
}

A. Kamyshnikova

Institute of engineering physic and radio electronics, Siberian federal university

Krasnoyarsk, Russian Federation

e-mail: komenzo@yandex.ru

\author{
S. Panko \\ Military engineering institute, \\ Siberian federal university \\ Krasnoyarsk, Russian Federation \\ e-mail:psp410@mail.ru
}

of operator errors. The result is a reduction in the testing quality and, consequently, an increase of the probability to miss products that are not correspond to the specifications, further operation of which leads to equipment failures. [3]

A partial solution to this problem is the use of automated test equipment (ATE).

Typical ATE complex allows to configure measuring equipment and to control measurement process in automatic mode, which minimizes the probability of operator error while setting up the measuring equipment [4].

However, such ATE does not fully eliminate the probability of operator error because the testing process is not completely automatic. Operator actions to install the necessary measurement parameters in accordance with a software test procedure, to launch the measurements in the desired order, to record the results of the measurements and to decide on their compliance with the specification remain manual.

\section{STRUCTURE DESCRIPTION}

Modern standards of information systems design provide a significant variability of equipment structure and characteristics, which significantly complicates the test 
procedure of different testing objects (TO) using a single typical ATE, because its abilities are limited to the functionality, inherent in the design process. This necessitates the creation of the programmable universal ATE, allowing to test TOs of various configurations.
To solve this problem, a hybrid testing equipment (HTE) was developed, which combines the advantages of manual and automated testing equipment [5]. The structural scheme of developed HTE is shown in Fig. 1.

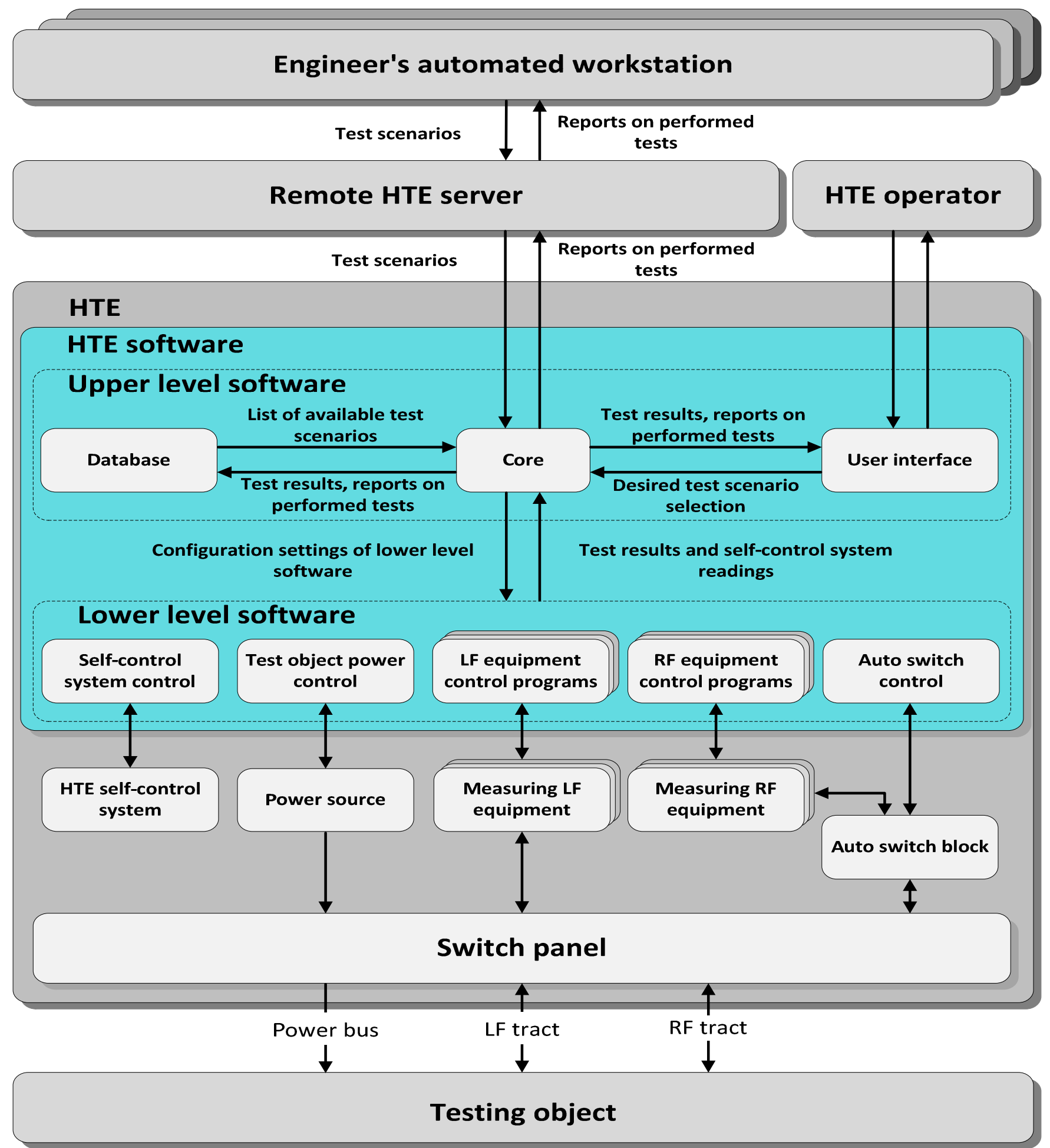

Fig. 1. The structural scheme of developed HTE 
The universality of designed complex is ensured by regular functions allowing to expand and modernize its functions in accordance with the tasks at minimal financial and time expenses. Increased functionality of HTE is exerised by adding new instrumentation and control software.

To enable the modernization of HTE by adding new control software a structure was developed, in which HTE is divided into two levels. Upper level is a single program while lower level is divided into sets of independent programs.

Upper level software is designed to interact with user and database and to control lower level software in accordance with a given test configuration, also to add new control programs in lower level software and to report on performed tests.

Lower level software is designed to interact with the hardware and consists of a set of independent programs to control the equipment and a number of utilities. Such a structure of lower level software makes it easy to expand the functionality of HTE adding new control programs or upgrading the old, in accordance with the tasks.

To add new control programs, standard functions of HTE upper level software are used, while the upper level software itself is not object to change. Upper level software launches lower level software programs in accordance with a given test configuration.

Testing procedure using HTE is divided into six stages:

1) Preparatory stage

The engineer interacting with HTE via remote server creates test scenario and saves it in the remote server database. Test scenario is a specialized file defining the scope and sequence of actions performed by HTE for measurement the characteristics TO and determination their compliance with the specification. Test scenario is created by operator by specialized interface which is part of the HTE software. The basis of the test scenario is the test program - the document that sets the object and purpose of the tests, types, sequence and scope of the experiments.

To cutting time which spend on the formation of the test scenario in the process of corresponding interface of HTE software is provided a multiuser mode. The group of operators has ability to work simultaneously on the test scenario.

2) Connection of the TO to HTE

The operator connects TO to the HTE switch panel and launches HTE.

Immediately after launching HTE synchronizes its database with the remote service database.

3) Download of required test scenario

The operator selects and executes required test scenario via UI (user interface) within upper software. UI data are being transferred to the upper level software core. Upper level software core downloads required scenario from the HTE database and defines which HTE devices will be used. Then upper level software core transfers equipment list, which will be used to execute test scenario, to control program of selfcontrol system within low level software.

Control program of the HTE self-control system diagnoses the necessary devices and also monitors environmental conditions and transfers the self-test results to upper level software core. After getting and analysing the self-test results, upper level software core launches the test scenario which was previously downloaded.

\section{4) Testing}

Upper level software core, designed to provide interaction between database, UI and lower level software, defines, which device (or combination of devices) and with which settings must be engaged at the moment, and also which limit values the test result must meet. In accordance with that, upper level software core launches programs, that control LF or RF equipment within lower level software, and then transfers required configuration settings to them.

Launched control programs read configuration settings, tune measuring equipment in accordance with that settings and with their purpose and afterwards start the measurement process.

Test results are being read by control programs, processed and transferred to upper level software core. Upper level software core defines whether these results correspond to the specification, displays them to the UI and also reserves them by duplicating in the HTE remote server database and in its own database.

The operator monitors the testing process via UI within upper level software and interferes with the process only in case of emergency.

For information, all of the data stored in HTE server databases can be discharged through the local network of the enterprise directly to automated workstation (AWS). At the same time the operator has the ability to control remotely the testing process in real time. Also, the operator has the ability to view not only the results and telemetry of tests carried out earlier, but use the built-in HTE software simulator, which allows to recreate the testing process per-second, what and in what order impacts were served, what parameters were controlled, what results were obtained, which reactions were occurred and so on. For reproducing the measurement process this simulator uses interface a similar with operator interface, but completed with a pause function, quick transition function and so on. The using of testing process simulator allows to analyze the results at a totally new level.

5) Test completion

Upper level software core generates a report on performed tests, that includes all the measurement results and other required data. This report is being saved in the HTE database, duplicated in the HTE remote server and transferred to the UI for display.

Report generating is a flexible process which allows to configure the final form of the report in accordance with the enterprise requirements.

6) Test results review

The engineer using AWS connects to the HTE remote server and downloads the report on performed tests to the computer within AWS. 
Data duplication in the HTE remote server allows to backup data and also allows the engineer to monitor the testing process from AWS in real time.

\section{CONCLUSIONS}

Described HTE structure has the following advantages:

1) The ability to create and add new control programs and measuring equipment in the process of HTE operation. It allows to expand functionality of HTE and to adapt it in correspondence to the tasks, which causes the universality of described HTE.

2) HTE self-control system. This systems allows to monitor the state of HTE devices and systems to minimize the probability of equipment failure during testing and also eliminates the possibility of testing using faulty equipment.

3) Auto switch system. This system allows to auto switch RF devices within HTE to the necessary RF inputs/outputs of the HTE switch panel without mismatch HF tracts. It also allows to automate the process of reconnecting TO's RF tract to various measuring devices.

4) Full function test of TO in automatic mode with minimal intervention of testing staff.

5) "Manual mode" function which allows to use HTE for troubleshooting in the product while debugging and adjusting.

6) Flexible process of automatic generation of reports of the tests carried out.

7) Ability a multiuser mode at the stage of testing scenarios.

8) Storage system with redundancy of them to a remote server and downloading data via a local enterprise network to AWS.

9) Possibility of remote work.

10) Testing process simulator.

The designed architecture of building a HTE combines the advantages of manual and automated test and control equipment (TCE), excluding a significant part of their inherent drawbacks. This architecture allows to achieve a high degree of automation of the test process not only, but also highly functional adaptability of the TCE in general.

Apart from deep automation HTE supports remote mode and multiuser access, and test process simulator opens up new possibilities in the field of the analysis of the results of tests carried out.

The ideology of simplicity of modernization and expansion of the functionality used in the design of this architecture allow increase greatly the versatility of the platform.

The reported study was funded by Krasnoyarsk Region Science and Technology Support Fund according to the research project: «Preparation for the introduction of the software and hardware complex for automation of tests of the on-board equipment of the tracking, telemetry and command subsystem in JSC "ISS"

\section{REFERENCES}

[1] A. A. Gorchakovsky, V. V. Evstratko, A. V. Mishurov, S. P. Panko, S. A. Ryabushkin, V. V. Sukhotin, V. A. Shatrov. Tasks and Their Solutions in the Process of Developing Control and Measurment Systems for Spacecraft // The Research of the Science City. 2015. №4. p. 6-9.

[2] Ai-Ping Li, Xue-Ping Li, Li-Guo Duan, Zheng-Hua Wang. Application Research on Legacy Software Reengineering in Automated Test System. // Journal of Applied Sciences 13 (8). ISSN 1812-5654 / DOI 10.3923/jas.2013.1227.1232.

[3] GOST R IEC 62508-2014 Guidance on Human Aspects of Dependability. -17.10 .2014 .

[4] S. P. Panko, A. V. Mishurov. Stages of the Design for Control and Test Equipment. Achievements of Modern Radioelectronics. - M.: Radiotechnics, №10, 2015.

[5] Patent RU2563925 (MPK B64G5/00), G01R31/00 - Control and Test Equipment / A. A. Gorchakovsky, V. V. Evstratko, A. V. Mishurov, S. P. Panko, S. A. Ryabushkin and others, JSC Academician M.F. Reshetnev Information Satellite Systems; Application number: RU2014118450/11 - 27.09.2015. 